\title{
Effects of frequency of prior incidental occurrence and recall of target words on anagram solution
}

\author{
MELVIN H. MARX \\ University of Missouri, Columbia, Missouri 65211
}

\begin{abstract}
Anagram solution was investigated as a function of the frequency of occurrence of target words in an immediately preceding, and presumably unrelated, word-completion task. Word recall in that task was found to be a negatively accelerated function of the frequency of occurrence. Anagram solution, however, was found to be inversely related to recall (recalled words were reliably less likely to be solved) and also, although not reliably, to the frequency of recurrence of target words in the prior task. These results are interpreted as suggesting that cognition, at least under some circumstances and as measured by recall, may be inversely related to transfer and, more generally, that cognitive factors need to be supplemented by noncognitive factors in the interpretation of learning and transfer.
\end{abstract}

The problem with which this experiment was concerned is the role of frequency of incidental occurrence, and subsequent recall, of target words prior to anagram solving in which the words are part of some of the solutions.

This work relates most directly to previous research both on incidental learning and on anagram solving. Frequency of incidental cue occurrence has been shown to be a significant determinant of recall (e.g., Cowan, 1980). In anagram research, one report (Dominowski \& Ekstrand, 1967) has indicated that prior presentation of semantic associates to target words can facilitate solution, although such associative priming was not confirmed in a later report (Jablonski \& Mueller, 1972). In both of these experiments, however, facilitation of anagram solutions was found when the priming list was composed of target words themselves.

\section{METHOD}

\section{Subjects}

Fifty high school students ( 30 female, 20 male) from two psychology classes participated.

\section{Tasks}

In Task 1, the subjects were instructed to fill in one-letter gaps in three- and four-letter common English words, so as to make complete words; they were given three trials on each of

This work was supported in part by a Research Career Award from the National Institute of Mental Health and a grant from the Army Research Institute for Behavioral and Social Sciences. The opinions expressed herein are those of the author and do not constitute endorsement by the U.S. Army. Thanks are due Joan Girnis, psychology teacher at Naples (Florida) High School, for making her two classes available for this research, which was conducted in accordance with approved safeguards. James Broyles assisted in preparation of test materials, and Kathleen Marx assisted in the collection and analysis of the data.
24 words (e.g., ti_e, _now, _ot). A 2-min free recall test was administered without advance notice for complete words after the third trial. Task 2 was then presented as a separate experiment; the subjects were given 48 anagrams to solve. Each anagram consisted of the scrambled letters of a common twoword phrase.

\section{Design}

Each of the 24 words presented in Task 1 offered an opportunity to complete a word that was subsequently part of a solution in Task 2 (e.g., tire for flat tire, snow for snow fall, hot for hot dog). Frequency of occurrence of these target words thus varied from zero to three, and this was a major independent variable; the other major independent variable was whether or not the word was recalled. Percentage of target words correctly unscrambled in anagram solutions for each of the frequencyrecall conditions was the major dependent variable.

\section{Procedure}

As earlier indicated, three trials of 24 incomplete words were first administered as Task 1. Each incomplete word was presented for $3 \mathrm{sec}$, with $2 \mathrm{sec}$ allowed for subjects to write in the completed word of their choice. Following the third trial, a previously unannounced 2-min free recall test of completed words was administered. Subjects were asked to write down as many such words as they could remember.

Task 2 was presented after a short rest period, during which record sheets were collected and new ones were distributed. Subjects were told that they were next to be given a series of scrambled words that they were to unscramble into familiar two-word phrases. Examples were provided. Thirty seconds were allowed for each anagram. In order to increase the solution rate, which otherwise would have been far too low to permit meaningful comparisons, the first letter of each of the two words in each anagram was written on the blackboard as clues. Only the target word was required for a response to be classified as a solution. The anagrams themselves, like the incomplete words, were presented by slide projector.

In order to equate the natural variability in ease of solution of the anagrams, the incomplete words presented to the first class related to one set of 24 anagrams and the incomplete words presented to the second class related to the other set of 24 anagrams. The two sets of anagrams were randomly distributed within the total list of 48 . The two classes were other- 
wise given identical treatment. Both tasks were presented during the regular $50-\mathrm{min}$ class period.

\section{RESULTS}

\section{Recall}

The percentage of recall for zero occurrences (false recalls, of course) through three occurrences of the key word was $4 \%(23 / 601), 21 \%(26 / 121), 35 \%(43 / 122)$, and $37 \%(126 / 341)$, for zero, one, two, and three occurrences, respectively. Owing to the large variability of score frequencies in the individual records, standard statistical measures could not be applied to these data.

\section{Anagram Solutions}

The most interesting results of the experiment are shown in Figure 1. This plot of the frequency of the percentage of anagrams solved as a function of prior incidental occurrence of target words, with separate curves for recall or no recall of key words, reveals two interesting relationships.

First, the consistent, and remarkably regular, superiority of the no-recall curve obviously suggests an inhibitory function for such recall. Although skimpy individual data again precluded a full ANOVA test, chi-square tests were applied to the individual scores for the most common occurrence value (three). With tie scores and incomplete scores omitted, 29 of 39 subjects solved a higher proportion of anagrams after having failed to recall a target word that had actually been given, in the word completion task, on all three trials. This proportion was reliably different from a chance 50-50 expectancy $\left(\chi^{2}=9.06, p<.01\right)$.

Second, the fact that the point of maximal targetword solution was the same for both recall and norecall curves, occurring in each case for the singlefrequency category, suggests an inhibitory rather than facilitatory function for frequency, once a single occur-

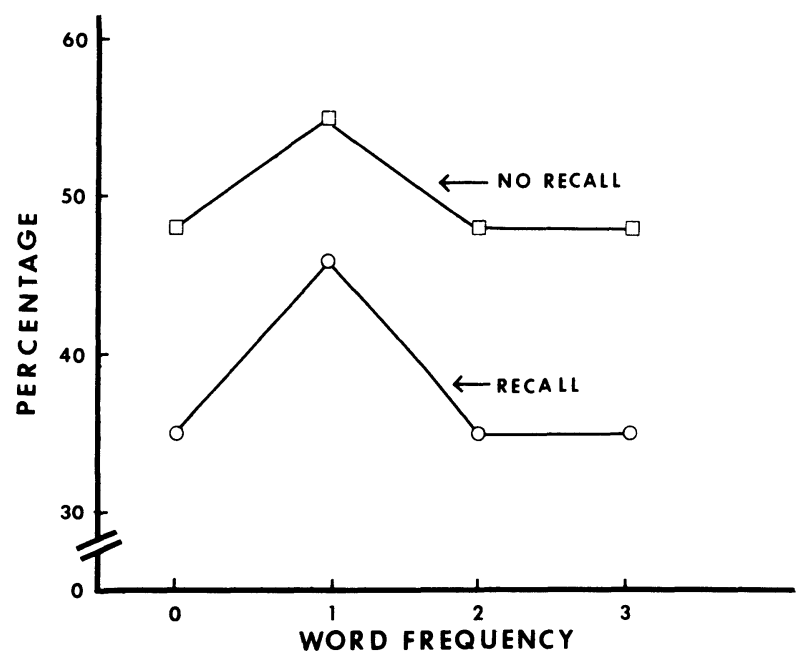

Figure 1. Percentage of successful retrieval of target words in anagram solution as a function of word frequency and of prior recall or no recall of target words.

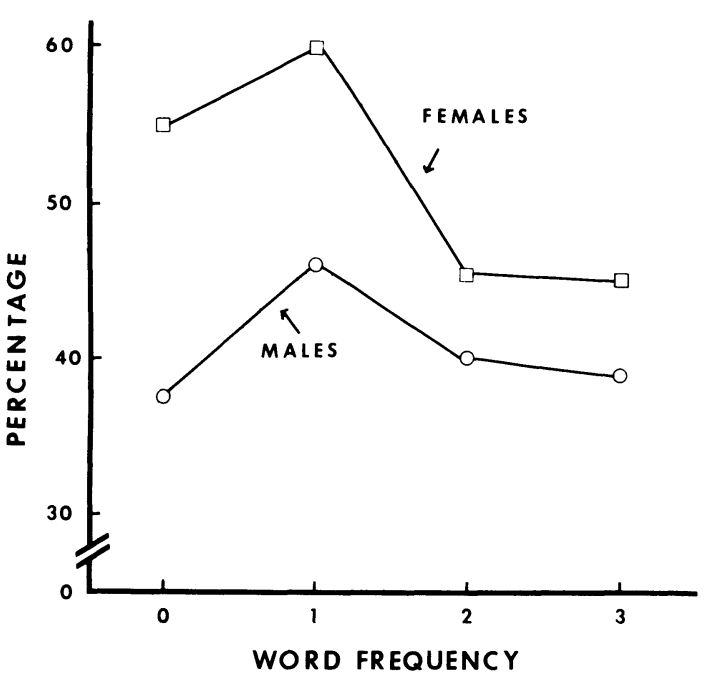

Figure 2. Percentage of successful retrieval of target words in anagram solution as a function of word frequency and of gender.

rence is achieved. Unfortunately, the skimpiness of the data at both the one-occurrence and two-occurrence categories (barely half of the 50 subjects could be scored) prevented an adequate statistical test; 17 of the 29 subjects whose one- and two-occurrence solution percentages could be contrasted showed a higher percentage for the former category, but this difference was not reliable $\left(\chi^{2}=.86, \mathrm{p}>.05\right)$.

That there was the usual priming effect, however, is indicated by the fact that 38 of the 50 subjects had a higher solution percentage after one or more occurrences of the target word compared with their anagram-solution percentage for the 24 control (no target word) items $\left(\chi^{2}=13.4, p<.01\right.$, assuming a $50-50$ chance baseline).

Figure 2 shows the percentage of target words successfully obtained in anagram solution as a function of prior frequency of target-word occurrence, separately for male and female subjects. Although both curves show the same general trend, it is clearly more marked for the superior female subjects. An attempt to test the apparent interaction was made by categorizing male and female subjects separately as to whether they solved a higher proportion of anagrams after one or two prior word occurrences. The interaction was evident, with 6 of 13 males and 12 of 18 females showing superior performance after a single occurrence, but the difference between these proportions was not statistically reliable $\left(\chi^{2}=2.54, p>.05\right)$.

\section{DISCUSSION}

The present results clearly confirm the priming effect in anagram solving that has been previously reported (Dominowski \& Ekstrand, 1967; Jablonski \& Mueller, 1972). However, the suggestion that there may be an inverse frequency effect from prior incidental exposure to target words and the more strongly indicated inhibitory effect of recall of such words raise in triguing questions. 
With respect to the recall effect, the first explanation that comes to mind is that the result may be due to something like the Zeigarnik effect (Zeigarnik, 1927/1938); in other words, completion of a task (in this case, the successful recall of a word) may have at least a momentary suppressing influence on the subsequent utilization of the response involved.

This phenomenon is seemingly related to Poetzl's (1917/ 1960) "law of exclusion" finding: Consciously noted features of pictures do not occur in subsequent hallucinatory reports (see Dixon, 1981, for an extended critical review of the Poetzl effect). In any event, whatever the mechanism, the apparent implication is that availability of a response in a transfer-like kind of problem solving is negatively related to its recent prior occurrence in a recall test. This result is clearly contradictory to the commonly reported finding, in conventional retention research, that repeated testing of recall in the absence of feedback serves to strengthen memory (e.g., Thompson, Wenger, \& Bartling, 1978; Tulving, 1962). For this reason, the clearly counterintuitive finding that prior recall can inhibit transfer cannot be expected to be widely generalizable. Nevertheless, because it suggests some potentially important and hitherto neglected relationships and interactions (for example, differentiation of recall and transfer), research designed to define its determinants and boundary conditions, as well as to establish its replicability, is indicated.

The Zeigarnik-type interpretation cannot be so readily applied to the inverse frequency effect, if indeed that apparent effect proves to be a replicable phenomenon. Because of the incidental character of the frequency variable, such an effect may require a different sort of explanatory mechanism. Future research directed at this problem might well manipulate the motivation variable (that is, use a standard learning task in place of or as well as an incidental learning task).

The suggestions of an interaction with subject gender (Figure 2), while not statistically supported in these data, nevertheless also seem worthy of further investigation. This interaction, if replicated, may be more reasonably attributed to the difference in verbal facility (i.e., female superiority) typically found to be associated with gender (Maccoby \& Jacklin, 1974). Differential facility, as evidenced by overall proficiency apart from gender, also needs to be carefully assessed in future research on the problem.

If the present design is viewed as a form of transfer of training, of the specific response variety, the inhibitory effects suggested in these data may constitute a serious restriction on positive transfer effects.

Finally, in spite of the rather unorthodox and unanticipated results obtained with the anagram-solution measure, the negatively accelerated recall curve (the decreasing increments in recall found as a function of increments in the more or less incidental exposures to key words) is consistent with the results of recent research on frequency of incidental stimulation and recall (e.g., Cowan, 1980). This orthodox result of the frequency variable may lend some credence to the less orthodox results, by its indication that the expected results on recall at least did occur. Moreover, and more important, the juxtaposition of these diverse results suggests that the same empirical operation can have diametrically opposed effects upon what may appear, superficially, at least, to involve highly similar processes, namely, recall and problem solving (or transfer). This point is important in light of the widespread recent tendency to equate cognitive and "learning"-response-selection-functions. The present results, if replicated, would certainly raise a serious question about any such assumption. They would thus indirectly support the contrary position (Marx, 1981; Marx \& Marx, 1980) that cognition (defined as knowledge, measured as recall) is only one of the important determinants of learning and should not be assumed to be equivalent to it.

\section{REFERENCES}

Cowan, R. E. Effect of instructions-to-learn and repeated semantic processing on long-term free recall. American Journal of Psychology, 1980, 93, 459-465.

Dixon, N. F. Preconscious processing. New York: Wiley, 1981. Dominowski, R. L., \& Ekstrand, B. R. Direct and associative priming in anagram solving. Journal of Experimental Psychology, $1967,74,84-86$.

Jablonski, E. M., \& Mueller, J. H. Anagram solution as a function of instructions, priming, and imagery. Journal of Experimental Psychology, 1972, 94, 84-89.

MaccoBy, E. E., \& JACKLIN, C. N. The psychology of sex differences. Stanford, Calif: Stanford University Press, 1974.

MaRX, M. H. Habit activation in human learning. In G. d'Ydewalle \& W. Lens (Eds.), Cognition and memory: Essays in honor of J. Nuttin. Hillsdale, N.J: Erlbaum, 1981.

Marx, M. H., \& MARx, K. Repetition of errors in learning and memory as a function of their prior associative strength. Bulletin of the Psychonomic Society, 1980, 16, 435-438.

Poetzl, 0 . The relationship between experimentally induced dream images and indirect vision. Psychological Issues, 1960, 2(Whole No. 7), 41-120. (Originally published, 1917.)

Thompson, C. P., Wenger, S. K., \& Bartling, C. A. How recall facilitates subsequent recall: A reappraisal. Journal of Experimental Psychology, 1978, 4, 210-221.

Tulving, E. Subjective organization in free recall of "unrelated" words. Psychological Review, 1962, 69, 344-354.

ZEIGARNIK, B. Das behalten erledigter und unerledigter handlungen. Psychologische Forschung, 1927, 9, 1-85. [Translated and condensed as "On finished and unfinished tasks" in W. D. Ellis (Ed.), A source book of Gestalt psychology. New York: Harcourt, Brace and World, 1938.]

(Received for publication March 12, 1982.) 